\title{
Las puertas de la memoria: Concepción (Chile)
}

\author{
Hugo Capellà Miternique ${ }^{1}$ \\ Recibido: 10-08-2020 | en su versión final: 20-10-2020
}

Resumen

\begin{abstract}
Concepción aparece como una ciudad nueva con pocas huellas referentes a su pasado, debido a una historia que se escribe a episodios de réplicas de terremotos. La ciudad chilena aparece siempre como un decorado nuevo, con pocas alusiones a un pasado atormentado que rima con superaciones colectivas. No obstante, las puertas y zaguanes de las casas representan una declarada excepción, anónima ante el transeúnte distraído. El presente artículo es uno de los resultados de una investigación científica, desde la perspectiva de la geografía cultural y de la arquitectura vernácula, realizada a partir de un trabajo etnográfico urbano. El interés es teórico a escala local, en la recuperación de la tradición urbana mapuche, así como, por otro lado, a escala más general, sobre la revalorización de la transmisión de un patrimonio vernáculo, como forma de resiliencia colectiva ante los desastres naturales. La costumbre de mantener o recuperar las puertas de las casas a lo largo del tiempo, no sólo es un hecho que obedece a una funcionalidad, sino que encierra un testimonio intencional, con una significación. Detrás de la conservación de la puerta original, hay la búsqueda de una continuidad, la hazaña de hacer patente una presencia y una identidad propia labrada a base de superaciones. La puerta es el símbolo como veremos de la permanencia y de la transmisión colectiva de una identidad y a la vez testimonio de reencuentro con el rico pasado cultural de raíz rural, europea y mapuche.
\end{abstract}

Palabras clave: referente colectivo; urbanismo; arquitectura vernácula

Citación

Capellà Miternique, H. (2021). Las puertas de la memoria: Concepción (Chile). ACE: Architecture, City and Environment, 15(45), 9643. DOI http://dx.doi.org/10.5821/ace.15.45.9643

\section{The Doors of Remembrance: Concepción (Chile)}

Abstract

Concepción appears as a new city with few traces of its past, written by episodes of earthquake aftershocks. The Chilean city always appears as a new setting, with few allusions to a tormented past that rhymes with collective overcoming. Nevertheless, the doors and hallways of the houses represent a declared exception, anonymous to the distracted passer-by. This article is one of the results of a scientific investigation, from the perspective of cultural geography and vernacular architecture, carried out, on the basis, of an urban ethnographic work. The interest is theoretical on a local scale, in the recovery of the Mapuche's urban tradition, as well as on a more general scale, on the revaluation of the transmission of a vernacular heritage, as a form of collective resilience in the face of natural disasters. The custom of maintaining or recovering the doors of the houses over time is not only a fact that obeys a functionality, but it also contains an intentional testimony, with a meaning. Behind the conservation of the original door, there is the search for continuity, the feat of making visible a presence and an identity of one's own, worked out by overcoming. The door is the symbol, as we will see, of the permanence and collective transmission of an identity and at the same time a testimony of the reunion with the rich cultural past of rural, European and Mapuche's roots.

Keywords: collective referent; urbanism; vernacular architecture

${ }^{1}$ Dr. en Geografía, Profesor titular de universidad, Departamento de Geografía, Universitat de les Illes Balears (ORCID: 0000-0003-2945-6741, WoS ResearcherID: B-7483-2016). Correo de contacto: hugo.capella@uib.eu

ACE, 15 (4.5) CC BY-ND 3.0 ES | UPC Barcelona, España | Las puertas de la memoria: Concepción (Chile). 


\section{Antecedentes y metodología: La puerta como referente cultural}

El presente trabajo es resultado de un proyecto de investigación titulado "Concepción: una tradición arquitectónica popular escondida detrás de las fachadas formales". Los retos de este proyecto realizado entre 2012-2014 en la Universidad de Concepción (Chile) fueron, por un lado, demostrar la importancia de los bagajes y experiencias domésticas por parte de la población local, como una forma de resiliencia ante un medio muy sísmico, como es Chile. Por otro lado, y no menos importante, se quería también aportar desde la geografía cultural y dentro de un equipo pluridisciplinar entre urbanistas, geógrafos y arquitectos una mirada en un mundo académico muy elitista que acostumbra a negar la existencia de un conocimiento vernáculo, por ser poco científico (Roderick, 1983).

El trabajo del grupo partió de un estudio etnográfico exhaustivo de paisaje urbano (Clay, 1987), por todo el centro de la ciudad de Concepción, para la recopilación de información hasta entonces inédita sobre formas de construcción domésticas. La investigación permitió evidenciar une serie de adaptaciones que se tipificaron (por ejemplo: doble-fachadas, fachadas-verja, doble-casa) y sirvieron como categorías para organizar el fondo fotográfico realizado (200 fotos). La documentación sirvió, por un lado, para testimoniar un legado vernáculo que se encuentra en proceso de desaparición, así como por otro para poder analizar de forma comparada cada tipología. El objetivo del análisis no era, desde un punto de vista cuantitativo, plasmar la distribución espacial, sino cualitativamente centrado en comprender el origen y construcción y significado del legado vernáculo, desde sus propios protagonistas. El trabajo fue, por ello, complementado con una serie de entrevistas con los vecinos y propietarios de esas casas (50), para conocer más de cerca, las motivaciones, lugares de aprendizaje, así como detalles sobre las formas construidas. Los primeros resultados de esta investigación, en lo relativo a las formas y las fachadas ya fueron presentados en el 54 Congreso IFHP, en Porto Alegre, en 2010 y publicados en 2014 (Capellà, 2014) y sirven como sustento para la continuidad de las investigaciones, en aspectos inéditos más particulares, como es el caso, ahora de las puertas.

Este elemento común, casi anónimo en general, representa en el caso de Concepción una de las únicas evidencias materiales para la articulación patrimonial colectiva en un contexto sísmico. La originalidad del enfoque del presente estudio versa, por un lado, en el enfoque cultural, así como por otro lado en la dimensión muy micro-local de estudio (a nivel de casa). El estudio de las puertas en Concepción se plantea desde una perspectiva socio-cultural al entenderse que representan un elemento simbólico en su adaptación cultural en un medio sísmico, así como en una adopción cultural histórica urbana, de un legado rural y mestizo (criollo y mapuche). A su vez, la dimensión micro-espacial de estudio, responde a la dimensión metodológica etnográfica y por lo tanto de corte más cualitativo (trabajo etnográfico, entrevistas). El objetivo no es tanto el de cartografiar una distribución, sino más bien el de poder demostrar el vínculo de la puerta con la representación colectiva, así como su vínculo cultural histórico. Esta priorización del segundo aspecto responde, a su vez, a la lógica de un patrimonio donde lo material, por lo general, pasa a segundo plano (Louvel, 2007) y en donde, por el contrario, se revaloriza, el aspecto de la transmisión del legado, como la única forma de permanencia cultural. En este contexto sísmico lo material pasa a ser tradicionalmente efímero, mientras que lo efímero de la transmisión oral termina convirtiéndose en lo relevante para la trasmisión colectiva. La labor en la investigación de recopilación de los testimonios a partir de las entrevistas, no sólo nos permite verificar la vinculación simbólica, sino que también se convierte en parte del mismo legado. La puerta pasa a convertirse en el único elemento físico que refleja el vínculo con el legado inmaterial y con la transmisión de unos conocimientos constructivos (vernáculos) que se convierten en un referente colectivo compartido.

\subsection{La memoria de la puerta}

ACE, 15 (4.5) CC BY-ND 3.0 ES | UPC Barcelona, España | Las puertas de la memoria: Concepción (Chile). 
Entre los elementos arquitectónicos que más apego despertaban entre los vecinos, se encontraba la puerta. La conservación de este elemento no sólo obedece a razones prácticas, sino que se convierte en un elemento de referencia colectiva (Capellà, 2010). En la ciudad de Concepción la puerta representa un elemento patrimonial que nos permite entender culturalmente toda una forma de vida. La puerta se convierte en el umbral a partir del cual reconstruir no sólo la vida material de la casa, sino también el hogar. La puerta marca la primera piedra angular sobre la cual se vertebra la resiliencia colectiva penquista (Capellà, 2013). La superación colectiva de los habitantes frente a los eventos sísmicos toma a la puerta como si fuera una plancha de salvación práctica, pero sobre todo desde el punto de vista simbólico como continuidad. Bajo la aparente sobriedad de la puerta, se encierra en cierto modo una parte del alma y forma de ser penquista.

A la hora de abordar este tema, nos encontramos con un profundo vacío bibliográfico, tanto a escala general, por ser un tema en la bisagra disciplinaria entre arquitectura, urbanismo, geografía, antropología y sociología, como a escala regional, por la falta de antecedentes sobre el tema específico entorno la puerta. El presente artículo, tanto por las metodologías utilizadas de trabajo etnográfico urbano, como por la temática abordada muy vinculada con el patrimonio y la memoria, nos han conducido hacia un enfoque más cercano a la geografía cultural. La escuela de Berkeley (Sauer, 2008) se basó en el análisis histórico y a micro-escala para una interpretación de los paisajes en clave cultural. Sus autores se centraron en el inventariado de los elementos arquitectónicos del paisaje rural americano, para poder establecer áreas de influencia culturales. Así, por ejemplo, en los trabajos de discípulos de Sauer, como Hahn o Butzer se inventarió la distribución del tipo de cercado (de dos o tres púas), la tipología de color de pintura de las granjas (rojas o bancas) o incluso el tipo de cierre de los cercados. Gracias a esos trabajos se pudieron llegar a sistematizaciones como la metodología de análisis del paisaje, planteada por Meinig y Jackson (1979). El uso del trabajo de campo y etnográfico como instrumento establece puentes con el presente estudio, aunque su propósito es parcialmente diverso. En los estudios de la Escuela de Berkeley el objetivo era poner de manifiesto las áreas culturales de los colonos de origen europeo en función de las formas de construcción de sus lugares de origen principalmente, de origen germánico, sueco, inglés o escocés. Por el contrario, en el presente estudio, el foco está centrado en la comprensión del proceso de construcción de las formas de adaptación cultural ante un medio sísmico, como resiliencia colectiva. La ciudad de Concepción ha documentado 9 terremotos, considerados según mediciones más recientes como mega-terremotos de más 6.5 en la Escala Richter. La ciudad y sus habitantes han tenido que adaptarse a una recurrencia media de 2 eventos por siglo en 1579, 1657, 1687, 1730, 1751, 1835, 1939, 1960 y 2010.

A pesar de todo existen puntos de encuentro entre los casos de estudio puesto que tanto en el caso de los colonos de Estados Unidos de América como en el caso penquista (habitante de Concepción), detrás de las formas se encierra un mismo planteamiento cultural centrado en el rescate de un patrimonio, entendido como un refugio para el referente. En el caso de los colonos, las formas vinculadas con el país de origen eran fruto de un bagaje práctico, pero a la vez marcaban un sello en la transmisión del eferente colectivo en las nuevas tierras. En el caso penquista, las formas también son un bagaje de experiencias colectivas transmitidas que representan un patrimonio más allá de las formas concretas. No obstante, a diferencia del caso anterior, se manifiesta una adaptación a un medio natural y a un sincretismo de las experiencias culturales de una población mestiza. La adaptación al medio natural como forma de supervivencia significó el reconocimiento indirecto de formas y tradiciones culturales de pueblos autóctonos. Los Mapuche habían convivido en ese medio desde hacía mucho más tiempo y desde una consideración cultural diversa respecto a la naturaleza entendida como parte de ella y no ya como oposición (Descola, 2012).

El estudio sobre la consideración de la puerta pone en evidencia el testimonio empírico de una tradición silenciada. Bajo la aparente adaptación funcional encontramos una inmersión cultural del bagaje patrimonial mapuche dentro del nuevo referente urbano penquista (Aravena, 2002). Las

ACE, 15 (4.5) CC BY-ND 3.0 ES | UPC Barcelona, España | Las puertas de la memoria: Concepción (Chile).

DOI: http://dx.doi.org/10.5821/ace.15.45.9643 
fuerzas telúricas forzaron a esa adaptación cultural. Las formas arquitectónicas europeas (Campos, 1985) tuvieron que adaptarse en un mundo, donde el referente fijo del suelo pasa a ser circunstancial al estar vivo, bajo el peligro sino de sucumbir (como sucedió de forma reiterada en los distintos terremotos que asolaron el centro y casas nobles decimonónicas del centro penquista).

\subsection{Abriendo puertas...}

Este caso de estudio permite plantear, más allá del interés local, un debate cultural abierto entorno a las formas de transmisión como patrimonio vivo. A su vez, pone de manifiesto la recuperación cultural mapuche debajo un aparente urbanismo anónimo y contemporáneo penquista. El presente estudio permite una doble revisión académica. Por un lado, resitúa temporalmente el rol mapuche en la cultura urbana chilena (Kropff, 2004). Los estudios sobre los Mapuche se centraban tradicionalmente en su vinculación con el medio rural. No obstante, más recientemente se ha ido ampliando la investigación al incluir desde una dimensión más sociológica (Aravena, 2003) el movimiento de autoafirmación de los jóvenes mapuches residentes en barrios periféricos de grandes ciudades.

El presente trabajo abre una vinculación histórica de la cultura mapuche con el medio urbano, al considerar las influencias de esa cultura de forma indirecta, en las morfologías de las edificaciones urbanas de los migrantes procedentes de zonas rurales. La huella de la cultura mapuche se encontraría de forma directa o indirecta en cualquier tipo de espacio. Este puede ser un gran aporte para los estudios, en clave chilena, al abrir una perspectiva cultural totalmente novedosa y en la cual encontramos otros casos potenciales afines, tanto en los territorios del Gran Norte de Chile con las culturas autóctonas Aymará, Atacameños, Changos, Diaguitas, como en el Sur, en el caso de Chiloé con Cuncos y Chonos o en Patagonia con Kawesqar, Yaganes, Selknam, Haush. El caso de la supervivencia de los galeses en Madryn gracias a su mestizaje con los Tehuelche, o el del bagaje Huarpe en Cuyo (Capellà y Benedetto, 2006) son un buen ejemplo para ilustrar unas realidades más comunes de lo que la historia escrita evidenció.

Por otro lado, el reconsiderar elementos arquitectónicos dentro del estudio geográfico retoma una larga tradición que había quedado silenciada por largo tiempo, al haber priorizado aspectos más vinculados con la representación. La revisión del legado de la Escuela de Berkeley permite poner de manifiesto una nueva relectura que pone de relieve las vertientes más teóricas y vinculadas con una visión ontológica de la cultura, en la configuración de sus representaciones. El enfoque del presente artículo permite en primer lugar volver a establecer puentes con la arquitectura en temas más allá del urbanismo (Hays, 1993). En segundo lugar, aporta una revisión crítica del legado de Berkeley (iniciada por Mikesell, 1978) desde una visión constructiva postmoderna que permite reabrir temáticas. El resultado de la incorporación de esta perspectiva en el estudio sirve a su vez como reflexión sobre la necesidad real de una transdisciplinariedad sobre temas que en una visión extremadamente compartimentada se pierden.

A su vez, el estudio aporta un interés práctico para el área de estudio al permitir el rescate de un legado popular, largamente ignorado y en serio riesgo de desaparición ante las nuevas lógicas urbanísticas (Pérez y Espinoza, 2008). Ese legado puede incorporar soluciones prácticas y sencillas desde criterios de sostenibilidad, medioambientales, así como de revalorización de las escalas locales. Encontramos investigaciones que revalorizan cada vez más el legado de las arquitecturas vernáculas en medios sísmicos como en Argentina o Irán, por ejemplo (Cirvini, 2011; Okhovat et al., 2011). Este bagaje patrimonial aporta respuestas adaptadas a realidades locales que empiezan a ser reconsideradas, incluso a nivel general. Así por ejemplo se empieza a ver en ciertas corrientes arquitectónicas alternativas, solidarias y de reciclaje (Elizabeth et al., 2005) la búsqueda de soluciones prácticas de bajo coste para mejorar la calidad de vida de los moradores. En el caso concreto de la

ACE, 15 (4.5) CC BY-ND 3.0 ES | UPC Barcelona, España | Las puertas de la memoria: Concepción (Chile).

DOI: http://dx.doi.org/10.5821/ace.15.45.9643 
puerta se empieza a considerar por ejemplo en caso de siniestros el reaprovechamiento de los cimientos de las casas, como una forma para abaratar costes y facilitar procesos de reconstrucción al permitir un más rápido encaje dentro de la cuadrícula y servicios urbanos preexistentes (Salvalai et al., 2017; Seike et al., 2018).

\section{El testimonio de la puerta}

La puerta es un elemento tan banal que pasa casi desapercibido en la ciudad. Toda casa tiene su puerta y se convierte para sus moradores, no sólo en un acceso funcional, sino también en el umbral entre el espacio personal interior y el exterior. La puerta es en cierto modo como la cara visible de uno, un emblema o como la cara visible de uno frente al otro. La propia numeración de la puerta fija la dirección postal y la identidad censal y del padrón. El elemento identificador espacial es como nuestro identificador individual (DNI). El número es la evidencia más clara de la importancia de la puerta como referente territorial que nos identifica de una forma funcional, pero, como veremos, también des de un punto de vista perceptual. La puerta se constituye por un lado como un hecho banal en su función cotidiana, pero por otro lado también se establece como un hecho particular en el cual cada familia residente volcará un esfuerzo e inversión, al ser la carta de presentación a su espacio interior.

La puerta desde ambas perspectivas se entiende como una marca que atestigua una presencia y significa un hito para cada familia. La puerta, por su relevancia referencial, se ha convertido en muchas culturas en un elemento de prestigio que se refleja en el uso de maderas nobles labradas y picaportes de metales nobles. Las puertas en las ciudades, además, se han convertido en muchas culturales en un elemento diferenciador en el caso de formas arquitectónicas muy homogéneas. Son una respuesta práctica para reconocer su propia casa y diferenciarse de la del vecino, aunque termina a su vez por convertirse en un emblema para definirse frente del otro.

La puerta ha terminado por convertirse en un elemento con entidad propia, desde un punto de vista particular pero también desde una perspectiva pública, contribuyendo a dar una entidad urbanística propia, incluso como reclamo turístico y patrimonial en ciertas ciudades como en Londres o Dublín.

\subsection{El silencio de las puertas}

El silencio detrás del anonimato de las puertas esconde los testimonios particulares de la mirada indiscreta de los transeúntes circulando por las veredas colindantes y representa a su vez, el ingreso cotidiano funcional de los moradores de la vivienda. Es el umbral de un ingreso al mundo más personal que se quiere salvaguardar de la mirada externa y a su vez es un elemento de significación, según atestiguan los comentarios en las entrevistas realizadas entre vecinos penquistas.

En el caso de Concepción las puertas constituyen uno de los pocos elementos patrimoniales de la ciudad a pesar de pasar casi desapercibidas. En una ciudad muy sísmica los elementos patrimoniales físicos son prácticamente inexistentes. La ciudad se ha ido reconstruyendo casi ex novo en cada ocasión, dando la sensación de novedad y poco apego de sus habitantes hacia un pasado que es asociado con desgracia (Oliver y Zapata, 1950; Pacheco, 1997).

En ese contexto en particular llama la atención la preocupación de sus habitantes en el mantenimiento de sus puertas como un elemento casi testimonial a pesar no demostrarlo públicamente, a tenor de las entrevistas. Es curioso detectar que esta práctica ha sido evidenciada y reconocida individualmente por los mismos habitantes a raíz de subrayar el tema con la presente investigación, pero no existía una real consciencia colectiva al respecto, siendo incluso motivo de sorpresa. El mantenimiento de las puertas se entendía más como práctica y costumbre particular sin

ACE, 15 (4.5) CC BY-ND 3.0 ES | UPC Barcelona, España | Las puertas de la memoria: Concepción (Chile).

DOI: http://dx.doi.org/10.5821/ace.15.45.9643 
ser conscientes que se trataba de una tradición colectiva. Su manifestación ha permitido recuperar un aspecto de una cultura popular. El factor de reconocimiento de la práctica es parte de un proceso más amplio de concienciación colectiva creciente por parte de los habitantes (Kyvig y Marty, 2000) que permite a su vez vincular el urbanismo desde una perspectiva social participante como parte y defensa de su patrimonio. Su largo silenciamiento, sin ser el motivo de fondo del presente artículo, puede obedecer a distintos factores como el largo proceso de represión política ante la libertad de expresión o la falta de reconocimiento des del mundo académico hacia las prácticas más populares y vernáculas, en un contexto de fuerte jerarquización social.

La puerta se convierte en el único elemento de continuidad en el tiempo entre la vivienda y sus habitantes. Marca un vínculo de arraigo de referencia individual pero también colectivo al considerarse dentro de un contexto urbano más amplio. Descubrimos como algunos vecinos se referencian dentro de ciertos espacios bastante homogéneos de la cuadrícula uniforme urbana de Concepción (Aguilera, 1994; García, 1996) a partir del color o forma de las puertas. El caso más conocido es el de un prostíbulo penquista que era conocido de forma anónima como el "portón verde" y que ha terminado convirtiéndose en un topónimo que forma parte de la memoria colectiva de los penquistas.

El valor agregado en los materiales nobles y trabajos realizados en las puertas son una de las causas funcionales para su reciclaje, así como para el mantenimiento de la antigua puerta en la nueva vivienda. Desde lo funcional hay que considerar la puerta, pero también el espacio ocupado que también se mantiene en los procesos sucesivos de reconstrucciones. La puerta marca en cierto modo la estructura interior y la distribución de la casa y en ese sentido su mantenimiento puede ser entendido de forma práctica como un reciclaje de la planta de la anterior construcción que retoma la puerta como referente re-articulador.

\subsection{Un noble testimonio}

La calidad de las maderas nobles chilenas usadas, como el alerce, el avellano, el ciprés, el coigüe, la lenga o raulí, junto con el trabajo de ebanistería, combinado con vitraux atestigua de unas puertas antiguas en un contexto urbano totalmente renovado. Si bien este tipo de puertas no representaría ninguna particularidad en otros contextos urbanos, en la especificidad penquista resaltan como uno de los pocos elementos materiales del patrimonio físico de la ciudad. Contrasta el ver puertas de finales de los siglos XIX e inicios del XX en formas arquitectónicas que, en el mejor de los casos, no tienen más de 50 años (como puede verse en las Figuras. 1, 2, 3 y 4). El resultado ante ojos ajenos es una incongruencia entre el conjunto moderno y una puerta antigua. No obstante, para la mayoría de los entrevistados, esta característica no es ni observada, porque forma parte de una realidad generalizada que es entendida casi como universal. Esta ambivalencia entre lo nuevo en la forma y un elemento viejo se aleja de toda lógica funcional si consideramos que una renovación debiera incluir todos los aspectos y aún más aquel que es más visible como la puerta. En consecuencia, este hecho responde a una motivación intencional. Tras diferentes consultas entre entrevistados se pasa desde la primera sorpresa por consultar sobre un aspecto que ni consideraban, a constatar el hecho en muchas puertas del centro penquista, y terminar por reconocer en algunos casos que también lo hacen en su propia casa. En esos últimos casos se pudo indagar más a fondo sobre las motivaciones. Tras la aparente falta de consideración reconocen que en muchos casos obedece a un propósito funcional ante la urgencia de las reconstrucciones post-terremotos, entendido dentro del reciclaje de elementos de la casa como las puertas (no sólo la de entrada) pero también, aunque en menor medida en ventanas (más endebles) y otros elementos menores como grifería. No obstante, al entrar más en detalle algunos entrevistados informan que se tiene mayor interés en recuperar las puertas de entrada de las casas y que incluso en los casos de una total destrucción se reaprovecha entonces incluso la de otra casa familiar, en caso de tener.

ACE, 15 (4.5) CC BY-ND 3.0 ES | UPC Barcelona, España | Las puertas de la memoria: Concepción (Chile).

DOI: http://dx.doi.org/10.5821/ace.15.45.9643 
Al entrar en el detalle, la mayoría de los entrevistados, aluden a que mantienen las puertas antiguas, "...por ser como lo habian visto hacer a sus mayores...," como parte de un bagaje transmitido de experiencias que son emuladas, por las nuevas generaciones al topar ante mismas situaciones. Recordemos que, con la mejora de las condiciones de vida, de media un penquista vivirá un par de mega-terremotos $\mathrm{y}$, por ende, puede tener recuerdo de episodios y respuestas anteriores transmitiéndose el bagaje de prácticas inter-generacionalmente. Esta actitud entendida como una respuesta individual tras las entrevistas pasó a tomar forma como una actitud colectiva, incidiendo en el carácter más cultural de la recuperación de las puertas.

De forma indirecta se fue poniendo de manifiesto, tanto por su carácter puntual,al afectar sólo a las puertas, como por su carácter más genérico como práctica colectiva que se trataba de una tradición cultural fruto de una adaptación cultural de influencias más. La puesta en valor del hecho a partir de la investigación sirvió de catalizador para qué la propia comunidad reconociera y tomara consciencia de una práctica patrimonial en una ciudad formalmente sin pasado material.

Figura 1. Ejemplo de la discontinuidad de elementos en las fachadas penquistas. Lientur 551

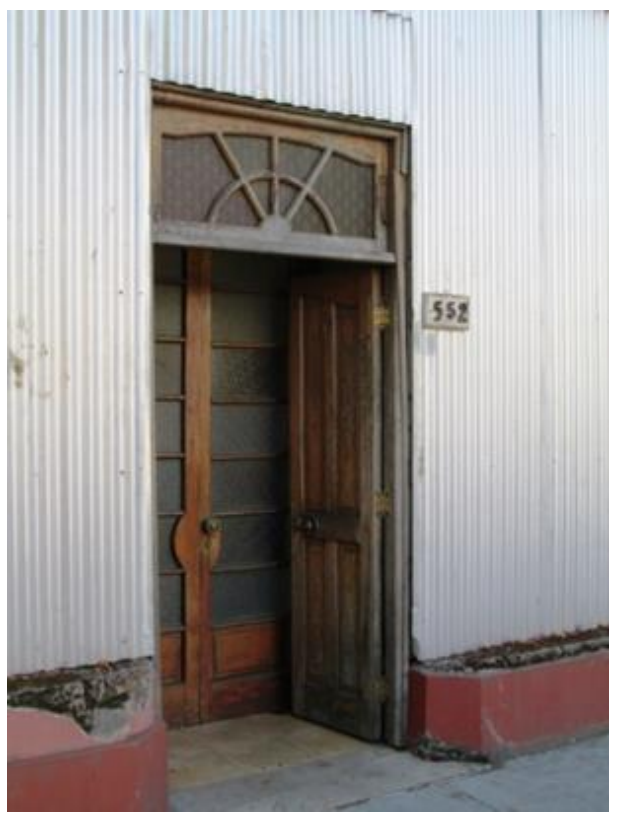

Fuente: Elaboración propia.

En el trabajo de campo se pudo constatar que la mayoría de las puertas atestiguan de las construcciones de principios del siglo XX con influencias modernistas y art déco tanto en el diseño de las puertas como en el uso común de zaguales, con puerta exterior más sobria y puerta interna más elaborada. La doble puerta y espacio intermedio genera una rutina casi ritual de teatralidad del interno particular con el externo anónimo en la rutina de abrir y cerrar la puerta exterior. Ese acto venía asociado tradicionalmente con el barrer la vereda o interactuar con la vecindad. El cierre del portón al final del día representaba de igual forma según atestiguaban los vecinos mayores el fin del día y el repliegue de los moradores en su interior. Además de generar una percepción de seguridad, el acto de cerrar guarda una dimensión simbólica importante, al marcar un ciclo de vida diario y anual en el cual la puerta y la casa se personalizan y adquieren casi un aspecto orgánico vivo (Eco, 1980). La vida de la casa sigue el ritmo diario de vida de sus residentes.

ACE, 15 (4.5) CC BY-ND 3.0 ES | UPC Barcelona, España | Las puertas de la memoria: Concepción (Chile).

DOI: http://dx.doi.org/10.5821/ace.15.45.9643 
De esta manera, la puerta por sus materiales, funciones, acciones marca un hecho en el cual la comunidad encontrará un símbolo sobre el cual anclar su permanencia, convirtiéndolo en un elemento patrimonial desde lo doméstico que va más allá de sus formas.

\section{La puerta: un testimonio}

La existencia de las puertas termina por convertirlas en un testimonio que permite la transmisión de un legado colectivo. El reciclaje de los espacios y puertas es una práctica vernácula común en Concepción que es legada y constituye una de las pocas prácticas patrimoniales visibles. Puede entenderse entonces como la necesidad colectiva de marcar urbanísticamente una continuidad en la ocupación de la vivienda como forma de autoafirmación de su identidad (Halbwachs, 1968). La puerta se transforma en un hito para sus moradores y un eje para una reconstrucción que si bien cambia la forma mantiene la continuidad del referente y su esencia en la permanencia de su puerta como un hito urbano (Lynch, 1960). Todo cambia, pero la puerta permanece como hilo conductor en el cual verter los afectos y sobre el cual constituir un apego a un lugar muy dinámico desde lo natural (Tuan, 1977). La puerta es la adaptación perfecta de una visión de asentamiento sedentario en un medio dinámico nómada (Capellà, 2013).

\subsection{Reciclando materiales}

Los procesos de reciclaje de los materiales post-desastre natural según los entrevistados y a vistas de las casas documentadas en el trabajo de campo son una práctica extendida que permite por un lado limpiar rápidamente los espacios para restablecer sus funcionalidades y por otro lado sirve como cantera de materiales para las nuevas construcciones. El conocimiento en las formas de reciclaje constituye una experiencia heredada de generación en generación. El proceso de reconstrucción y reciclaje de materiales constituye una experiencia colectiva reconciliadora en el proceso posttraumático, generando lazos sobre los cuales se establecen los cimientos de la identidad colectiva del lugar (Francis, 1982).

Figura 2. Las puertas se mantienen, a pesar de las transformaciones de la casa. Heras 1060
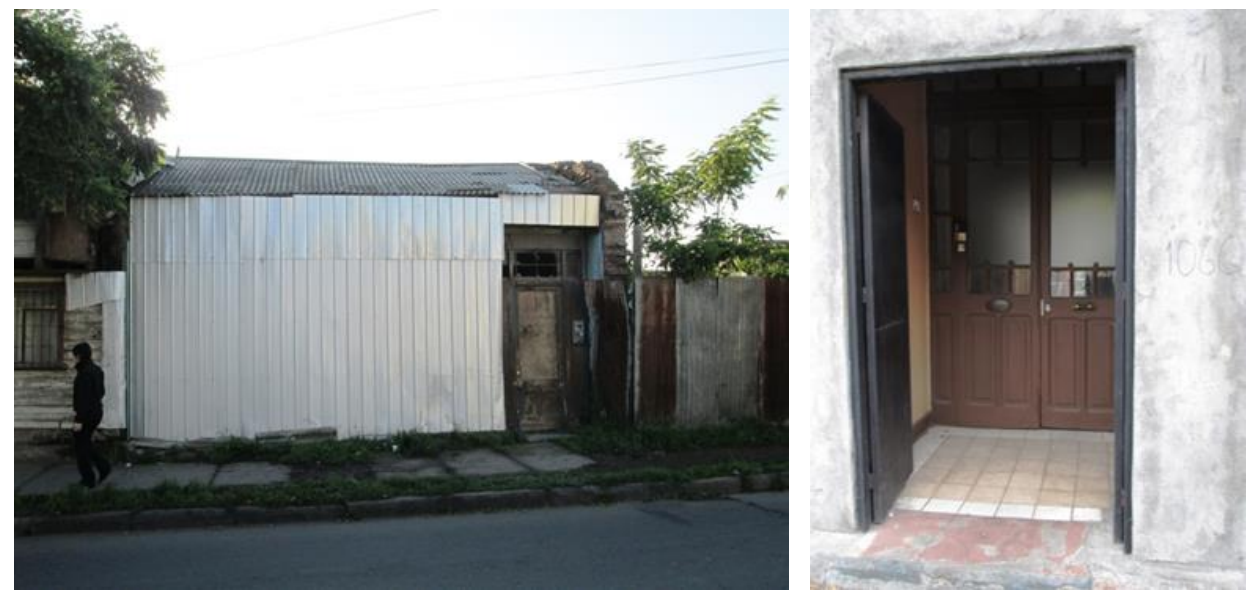

Fuente: Elaboración propia.

El reciclaje de materiales es común en los procesos de reconstrucción de las casas, sobre todo al alero del modelo de construcción de dos casas $(2 * 101 * 2)$ en materiales distintos como madera versus piedra que permiten una mayor resistencia y adaptación frente a los eventos telúricos (Capellà, 2014). En ese sentido cabe destacar la preocupación en particular por el reaprovechamiento de tres

ACE, 15 (4.5) CC BY-ND 3.0 ES | UPC Barcelona, España | Las puertas de la memoria: Concepción (Chile).

DOI: http://dx.doi.org/10.5821/ace.15.45.9643 
elementos debido a su coste tanto económico como de horas de trabajo. El reciclaje de los cimientos de las antiguas casas y los baños permiten ahorrar y guían en la reorganización de la reconstrucción de las nuevas viviendas. No obstante, en el caso específico de las puertas tenemos que considerar dos aspectos.

Figura 3. Aunque las fachadas caigan el espacio de la portada y la puerta se mantienen. Cruz 500

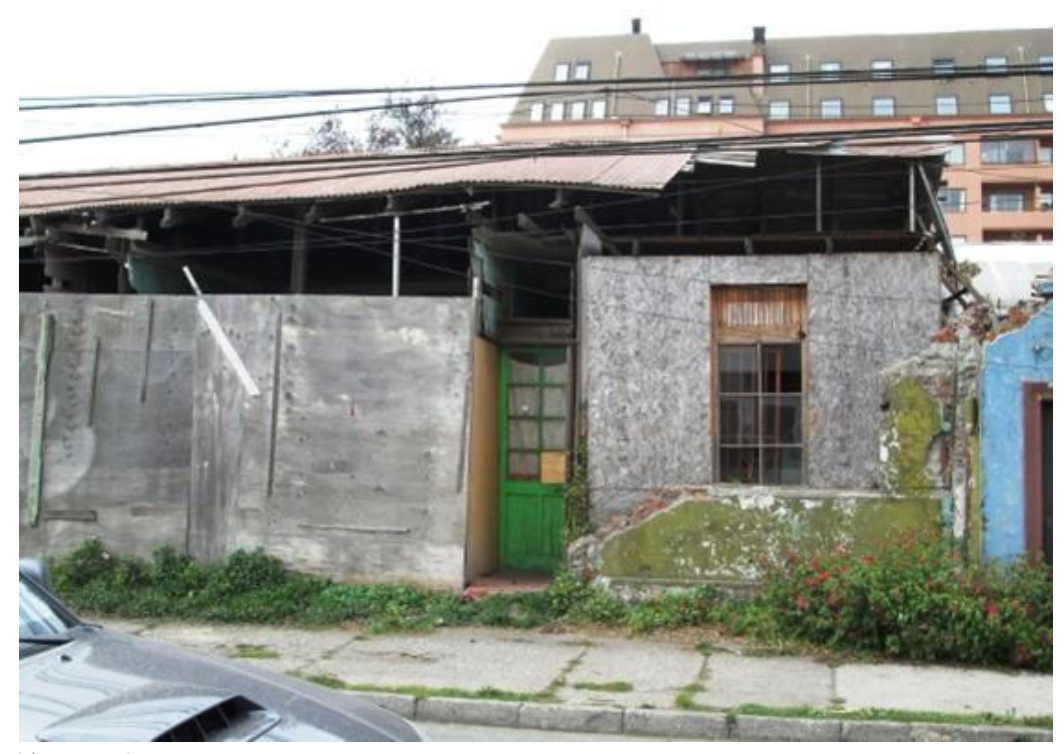

Fuente: Elaboración propia.

Por un lado, el espacio que ocupa la puerta o portada debe entenderse desde el punto de vista estructural de la configuración de todos los espacios internos de la casa. El retomar el mismo espacio para reconstruir una vivienda es una medida de ahorro similar en este sentido a la reutilización de los cimientos o de los baños, como atestiguan muchos de los entrevistados. Por otro lado, el valor económico de las puertas realizadas muchas veces en maderas nobles las convierte en un elemento reutilizado en la reconstrucción. Cabe añadir que la puerta es un elemento fácil de reinstalar. El resultado muestra la adecuación de puertas antiguas adosadas en nuevas estructuras de viviendas sin homogeneidad estilística y, además, en muchos casos con problemas de encaje, al tener proporciones diversas. Las puertas de casas antiguas de principios del siglo XX guardan unas proporciones de techos altos que no se adecuan a las proporciones más reducidas actuales. Ciertas puertas antiguas, reutilizadas en Concepción, terminan dando una impresión de falsas perspectivas entre zaguanes altos con puertas interiores levantadas y más pequeñas.

Sin embargo, el ahínco y trabajo por readecuar una puerta antigua en un espacio nuevo diferente (Figura 5) se aleja del sentido pragmático y práctico en la reconstrucción post-terremoto y evidencia, por el contrario, aunque sea de manera inconsciente o por costumbre, una práctica que responde a otros intereses de corte más psicológico, social y cultural. El valor concedido a la puerta, si bien obedece a unos motivos racionales y funcionales tales como el costo de las maderas nobles o su fácil reutilización, también obedece a una dimensión simbólica asociada a su valor (Cirlot, 1969)

La puerta representa la cara de ingreso a la vivienda y adquiere un carácter referencial. El mostrar una puerta elaborada y trabajada simboliza en cierto modo un status de cara al exterior. La puerta en ese sentido es también un símbolo de status social que se quiere mantener con su reutilización. La permanencia de la puerta se convierte indirectamente en uno de los únicos símbolos materiales patrimoniales que marca un testimonio de la continuidad de una familia y de su vivienda. Se

ACE, 15 (4.5) CC BY-ND 3.0 ES | UPC Barcelona, España | Las puertas de la memoria: Concepción (Chile).

DOI: http://dx.doi.org/10.5821/ace.15.45.9643 
transforma de forma casi involuntaria en un emblema de un legado que pervive en el tiempo, más allá de los desastres naturales recurrentes.

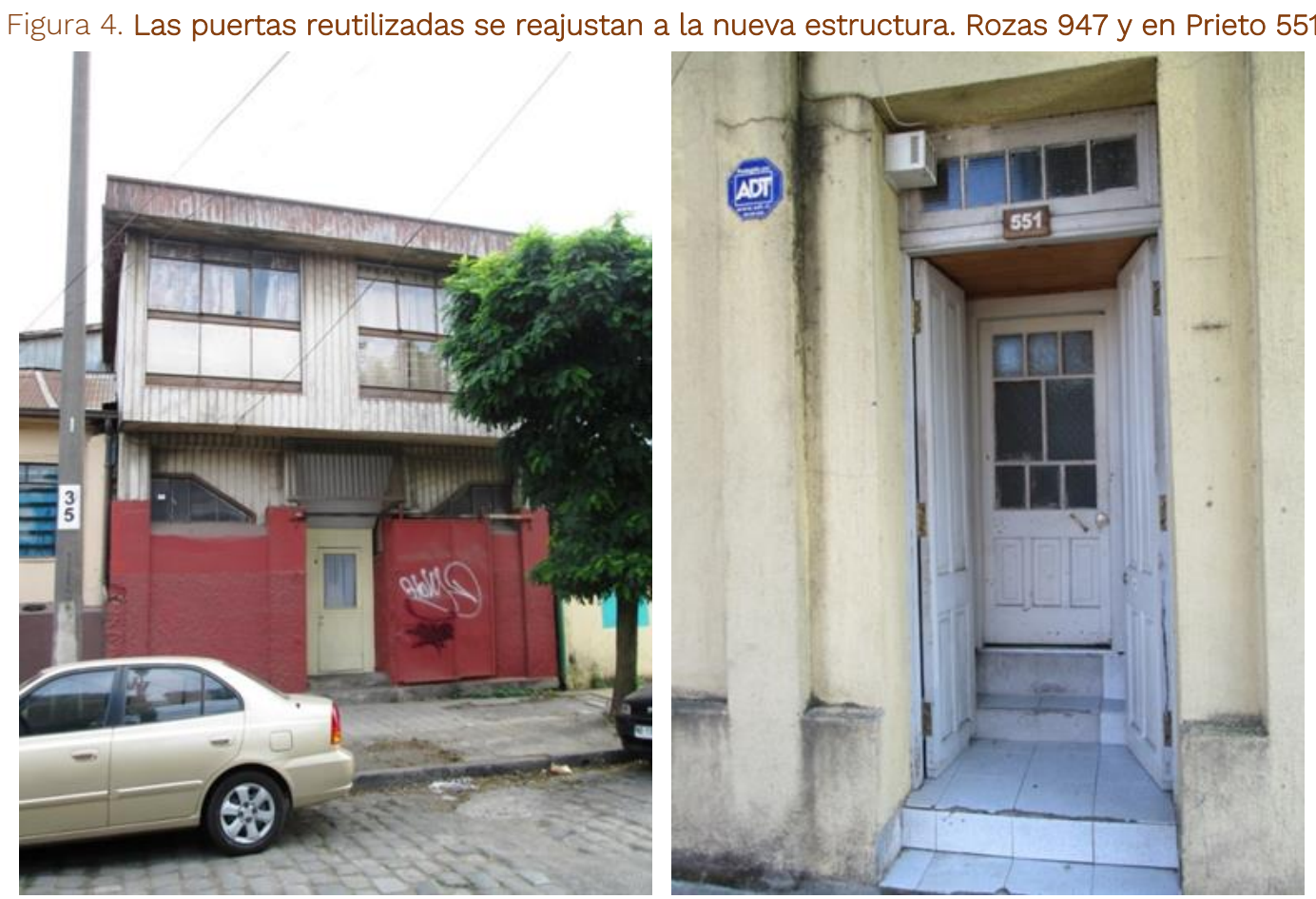

Fuente: Elaboración propia.

\subsection{La necesidad de continuidad}

De la misma manera espontánea y aparentemente sin razón que la gente en Concepción tiende a colgar fotos antiguas de la ciudad en sus negocios, las puertas pasan a constituir otro de los pocos elementos en alusión a un pasado y un patrimonio visible (Capellà, 2010). Lejos de ser una práctica constituida y consciente observamos cómo es en los barrios centrales más populares donde muchas de esas prácticas han pervivido y se han transmitido. Desde esa perspectiva nos encontramos con una población que valora las puertas porque atestiguan en cierto modo de la supervivencia de sus núcleos familiares y de sus viviendas. El conservar la puerta no obedece tanto a una lógica estética puesto que muchas veces estos testimonios del pasado rompen con las nuevas formas y materiales constructivos.

Las puertas en este caso son vistas como el testigo que permite la transmisión de un legado. Cada puerta encierra por un lado los espacios interiores que quedan encapsulados bajo el post-trauma, pero sobre todo por otro lado encierra todos los recuerdos del pasado vivido. En la puerta se concentra una vivencia y experiencia que quiere pervivir. La vigencia de la puerta simboliza, entonces, la continuidad de un referente colectivo adaptado a la realidad dinámica del medio local. A lo largo de las entrevistas la puerta se convirtió en una excusa para rememorar testimonios de vida y familiares de seres queridos, ya ausentes.

La importancia del mantenimiento de la puerta no es baladí. Recordemos que en la contrapuerta de muchas de ellas se encuentran; estampas religiosas, herraduras, cruces, cruces de mayo, espigas, palmas de ramos, coronas de navidad o muérdago, que simbolizan la protección para la familia que vive dentro, así como la bendición para el que sale fuera. La puerta es un umbral y, a la vez, un espacio sacralizado asociado al cristianismo, pero también reviste sincretismos anteriores. Para la

ACE, 15 (4.5) CC BY-ND 3.0 ES | UPC Barcelona, España | Las puertas de la memoria: Concepción (Chile). 
cultura mapuche la puerta de ingreso de cara al Este tiene también una relevancia espiritual y práctica, como veremos más adelante. La puerta reviste significados que van más allá de lo consciente, pero que han pervivido por tradición y transmisión.

Los entrevistados no son conscientes de toda la dimensión histórica, aunque sorprende su ahínco en mantener la tradición. Igualmente se sorprenden al averiguar que los demás también hacen lo mismo al evidenciar las otras puertas antiguas que para ellos forman parte de un paisaje cotidiano. No obstante, al poner el énfasis en la puerta empiezan a reconocer la importancia que tiene para ellos en lo afectivo y simbólico. El ejemplo de las puertas en Concepción sería el inicio de un proceso de reconocimiento de una cultura popular silenciada o entendida como obviedad irrelevante. Tal vez haya sido su olvido lo que haya permitido su pervivencia. No obstante, el ahínco en mantener esa costumbre familiar no deja de demostrar el interés inherente por un pasado. Los habitantes siguen realizando una práctica entendida como funcional, sin saber que están reproduciendo la continuidad de una práctica simbólica relevante ancestral. El poner de manifiesto la situación ante los entrevistados toma sentido para ellos y despierta un sentimiento de orgullo al haber sabido mantener un patrimonio y como primer paso hacia una recuperación de sus referentes.

\section{La puerta: un significado}

La puerta simboliza la continuidad dentro de la refundación. La puerta seguirá dando paso a las distintas generaciones a modo de una suerte de bendición. Sigue además la representación simbólica del ciclo anual de celebraciones, con distintos elementos como la cruz de mayo, la palma de ramos - la corona de navidad. La puerta es a modo de altar improvisado que nos protege del exterior desconocido y se convierte en cierto modo en un lugar improvisado y anónimo de culto, casi portátil. En la puerta se simboliza además el honor de la familia. Su nobleza refleja el rango frente al resto. La puerta como espacio de transición tanto desde lo interior al exterior como del exterior al interior representa el tránsito materializado. Es un ente que sintetiza la permanencia y termina por convertirse en hito referencial para el grupo humano que habita en esa vivienda (Corraliza, 1987).

\subsection{La refundación}

La puerta es normalmente uno de los únicos elementos que resiste incluso a un incendio (Figura 5). Su instalación cuando se construye una casa, marca el cierre o término del proceso. Cuando la puerta se instala, se establece conceptualmente la diferenciación entre el exterior e interior y se hace habitable como vivienda.

La puerta presupone un perímetro y cerco previo que se traza para diferenciar el interior del exterior. En la fundación de ciudades y fortalezas vemos como este proceso ha sido marcado desde la antigüedad con un profundo significado espiritual para aportar buenos augurios para el nuevo establecimiento. En un acto simbólico, tal como marcar el perímetro con un simple trazo, enterrar la primera piedra, celebrar la instalación de la techumbre se incluye finalmente la entrega de la llave de la puerta principal. La puerta simboliza en este sentido la propiedad y culminación con el cierre de un perímetro y de un proceso temporal (Conrad, 2010).

ACE, 15 (4.5) CC BY-ND 3.0 ES | UPC Barcelona, España | Las puertas de la memoria: Concepción (Chile). 
Figura 5. La puerta resiste al incendio y se transforma en el primer y último elemento de la casa desde un punto de vista simbólico

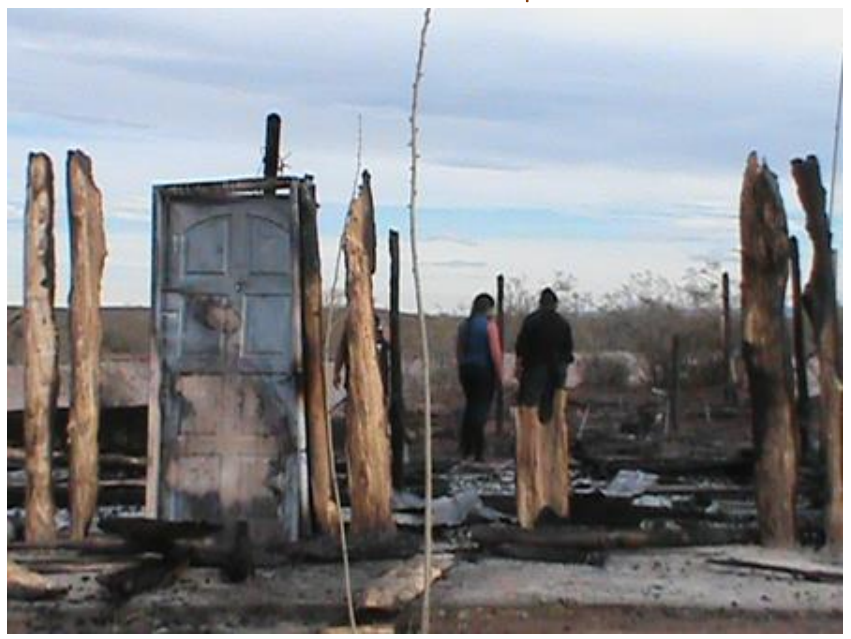

Figuras 6 y 7. La importancia simbólica de la puerta y del grupo familiar
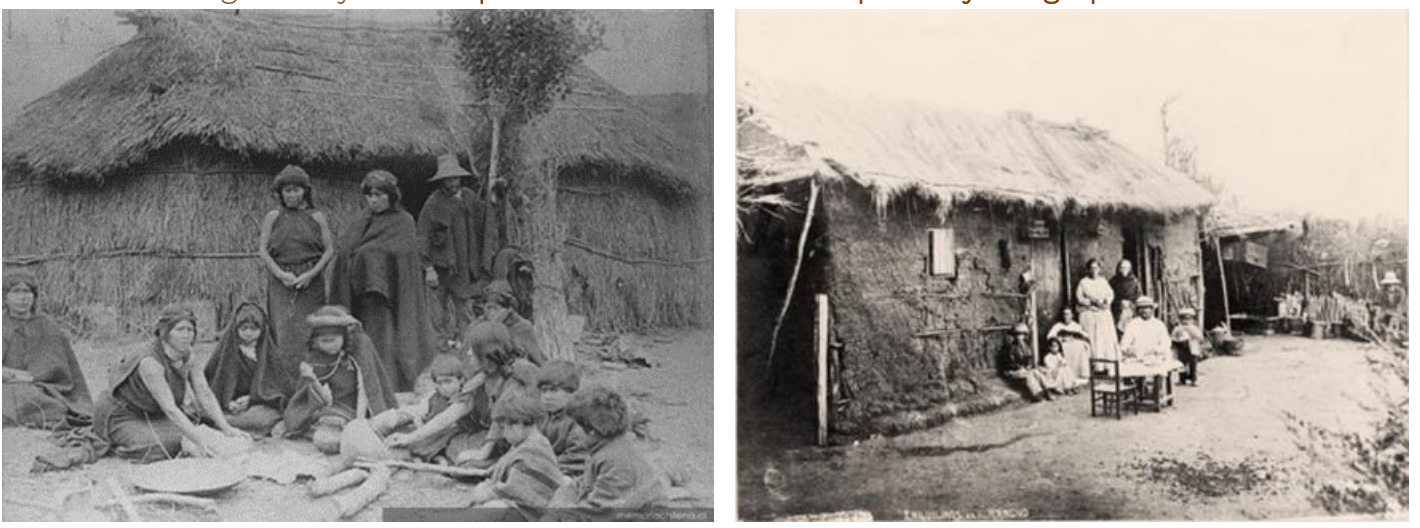

Fuentes: (Alvarado et al., 2001); archivo particular Barriga.

La reutilización de una misma puerta en una nueva vivienda marca una continuidad con la casa anterior puesto que a efectos simbólicos sigue siendo la misma. La reconstrucción en sentido simbólico no debería ser considerada entonces como una refundación al entenderse como un encadenamiento al mantenerse la puerta, la misma cerradura y llave (Savin, 1998).

Por más que la forma de la casa haya cambiado, su alma es la misma (Hakim, 2007). Esa identidad que pervive y se transmite permite a sus habitantes reencontrar en el referente del lugar, el eje de la continuidad de una identidad territorial pese al carácter dinámico y traumático provocado por su medio natural. Esa forma de adaptación termina siendo la identidad propia y es la que permite seguir viviendo en un lugar que otros considerarían como adverso (Cooper, 1971).

\subsection{La puerta como vínculo con el pasado}

La puerta de la vivienda urbana en Concepción a pesar de su formal modernidad refleja una larga tradición más compleja que podemos encontrar en la ruka mapuche (Figura 6), así como en los ulteriores ranchos rurales y luego urbanos (Figura 7) (Villalobos, 2010). Desde la visión tradicional mapuche, la casa (ruka) no tenía aperturas, con la excepción de la puerta que además guardaba por

ACE, 15 (4.5) CC BY-ND 3.0 ES | UPC Barcelona, España | Las puertas de la memoria: Concepción (Chile). 
un tema funcional y espiritual una orientación hacia el Este, vinculada con la luz solar y entendida como energía positiva. No es de extrañar que al lado de la puerta se acostumbrara a instalar tradicionalmente el Rewe, (tótem) que marca el vínculo con los ancestros. La puerta igualmente se consideraba como uno de los lugares más nobles de la casa. De ahí que las familias se tomaran las fotos (Figura 9), como un símbolo de orgullo y honor para manifestar el lugar e identidad de pertinencia (Tuan, 1974). El rancho rural y ulteriormente su versión adecuada a la cuadrícula urbana de las ciudades (Figura 8) retomará el modelo de la ruka mapuche pero reinterpretada desde las formas de la casa colonial. De esta manera nos encontramos con casas que tendrán pocas ventanas exteriores con una gran sala interior oscura y dando un gran protagonismo a la puerta central de la casa como único foco de luz.

La puerta pervive como lugar noble y emblemático donde las familias se toman sus fotos (Cooper, 1978). La tradición mapuche llega a la ciudad por medio de la adaptación del sincretismo criollo rural de forma indirecta. La relación urbana con la cultura mapuche (Solano, 1990) hoy muy proclamada desde ciertos ámbitos académicos en lo demográfico y social, tendría en algunas áreas del Sur de Chile, una larga conexión, aunque nunca evidenciada como tal. En este sentido una revisión de los estudios urbanos de ciudades como Concepción desde esa perspectiva cultural mapuche sigue pendiente y puede aportar luz sobre algunas de las prácticas y características urbanísticas chilenas, como por ejemplo en las (re)fundaciones europeas de asentamientos ya habitados que insertaron de forma sincrética las territorialidades previas. La ciudad chilena encierra el reencuentro de una nueva reivindicación mapuche en el medio urbano, la revisión del mundo criollo desde el sincretismo y el mestizaje, sin por ello negar los procesos clasistas y colonialistas.

\subsection{La puerta de la vergüenza}

La prevalencia de esa tradición cultural prehispánica se convirtió en un rico legado práctico transmitido en un medio natural desconocido y hostil para los nuevos colonos hispánicos. La arquitectura doméstica se convirtió en una forma silenciosa más de transmisión de un rico legado prehispánico, obviado. La relevancia ritual prehispánica acordada a la puerta se sincretizaría con otras tradiciones donde la puerta era ya más un referente de estatus social, como blasones o materiales nobles para marcar el estatus económico de la vivienda herederas de tradiciones previas en las culturas europeas y mediterráneas. El caso de las puertas en Concepción es un ejemplo más de los sincretismos producidos en América. No obstante, en el caso concreto chileno se establece como uno de los pocos elementos patrimoniales que ha pervivido a los embates telúricos. La transmisión de su legado se ha evidenciado en las fotos del artículo, despertando de su olvido. La invisibilidad de este elemento debe también enmarcarse en un profundo olvido por parte del mundo académico y en particular desde la tradición de escuela entre arquitectos que se traduce en un rechazo implícito por parte de ciertas élites hacia todo aquello vernáculo (Coquery-Vidrovitch, 1996). Se ha obviado un durante tiempo así un legado, una experiencia y una fuente de conocimiento que empiezan a ser reconsiderados ahora (Carter, 2005).

Las tradiciones en este contexto si son domésticas son ignoradas por lo general. El evidenciar el valor simbólico de la puerta y el legado que encierra desde un punto de vista práctico, pero también histórico se convierte dentro del contexto académico concreto latinoamericano, chileno y de provincia, en una verdadera reivindicación. Representa el merecido reconocimiento a gran parte de una población hasta ahora aún poco estudiada y silenciada. En este sentido cabe recalcar, por un lado, la gran dificultad en poder avanzar en la investigación, así como en la reacción por parte de ciertos sectores académicos adversos en querer evidenciar este rico legado, entendido como "sin relevancia patrimonial". Por otro lado, la investigación ha provocado la casi perplejidad por parte de los vecinos de Concepción al entender que su esfuerzo en el tiempo, encerraba un rico legado del cual tomaban conciencia y que les empoderaba.

ACE, 15 (4.5) CC BY-ND 3.0 ES | UPC Barcelona, España | Las puertas de la memoria: Concepción (Chile).

DOI: http://dx.doi.org/10.5821/ace.15.45.9643 
Figura 8. Las ciudades chilenas van a retomar la forma de vida dispersa rural del rancho adecuado a la nueva estructura urbanística de las cuadras, como parece en la foto del Sector poniente en 1913, en Concepción

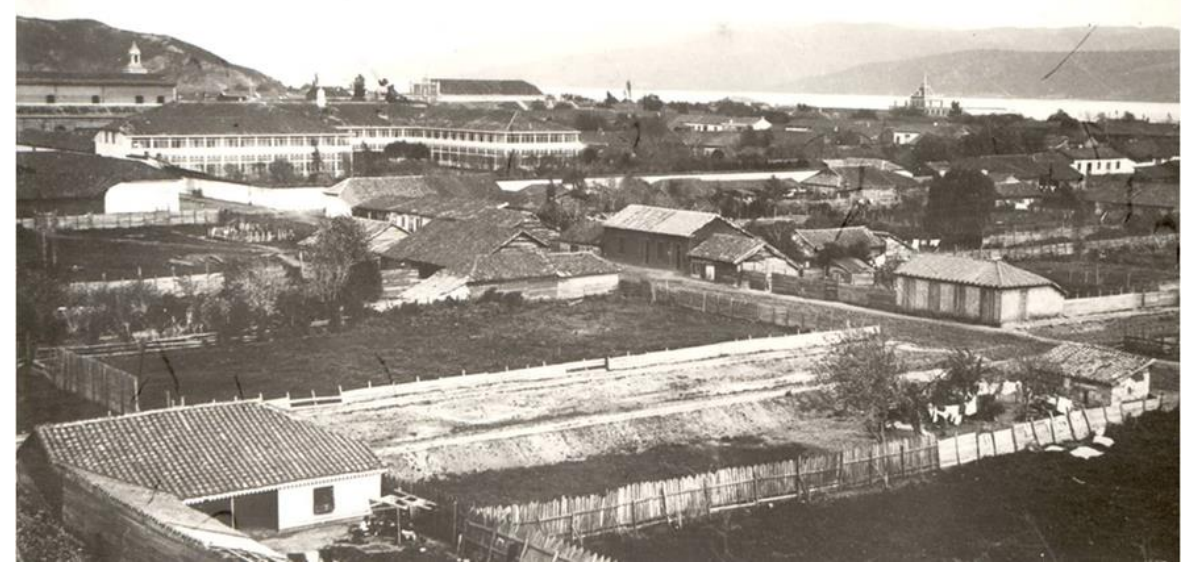

Fuente: Archivo Luis Darmendrail

Figura 9. La puerta es el símbolo y orgullo de la familia

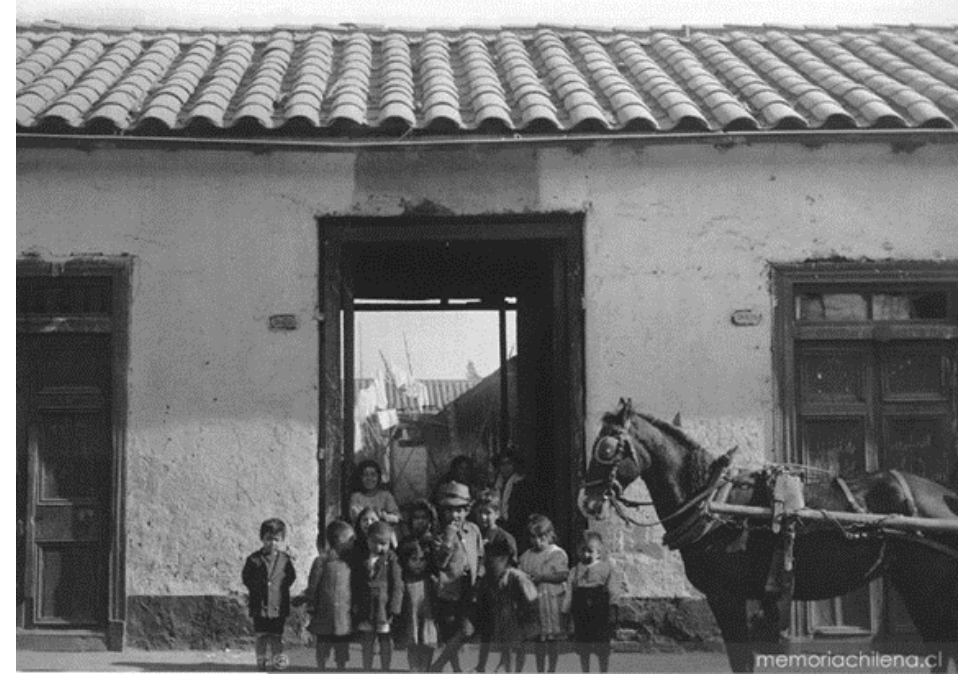

Fuente: (Chilectra, 2001)

\subsection{La puerta resiste}

Todos estos antecedentes subrayan la tradición multicultural implícita en la importancia atribuida a la puerta en el contexto urbano en Concepción. El conservar una puerta sería en el fondo una herencia de un pasado cultural del cual ya se desconoce casi su origen. La transmisión de la tradición y por causas funcionales habría sido la razón de su pervivencia. Detrás de un aparente hecho natural, se encerraría una larga construcción cultural e histórica. El mantenimiento de las puertas antiguas de las casas representa un legado patrimonial colectivo que ha pasado totalmente desapercibido bajo el manto uniforme de modernidad impuesta en las distintas reconstrucciones oficiales tras cada terremoto. Las puertas habrían sido el mejor recaudo para refugiar los recuerdos y una memoria urbana casi nómada. La puerta simboliza la grandeza de la historia de los fundadores, su tradición. En su transmisión, está la continuidad de su legado y su forma de vida (Nogué, 2007).

ACE, 15 (4.5) CC BY-ND 3.0 ES | UPC Barcelona, España | Las puertas de la memoria: Concepción (Chile). 
El caso de las puertas en Concepción dista de ser una excepción y encontramos en distintas regiones del mundo situaciones parecidas respecto a la relevancia de la puerta, más allá de su función. Así desde la yurtas mogolas (Xiao-Ju y Wu, 2009), pasando por las llaves que aún conservan como último vínculo referencial las familias descendientes sefardíes de sus casas que debieron abandonar al ser expulsados de España hace más de 500 años, son muestra de la importancia simbólica de una puerta (Savin 1998). Dentro de esa perspectiva es curioso observar la poca relevancia que se ha dado en los estudios tanto urbanos como en la geografía en general a este elemento de la casa. A pesar de ello, encierra un testimonio que puede encerrar mucha información en clave social, histórica y cultural. Las puertas resisten al paso del tiempo y se convierten en testigos valiosos tanto para el académico, como para sus moradores. Encierran un referente lejos de su primer anonimato y participan a su vez en la comprensión de un urbanismo entendido como una praxis colectiva de vida y no sólo como un diseño técnico.

\section{Agradecimientos}

Este artículo ha sido realizado con materiales derivados de un proyecto de investigación dirigido en la Universidad de Concepción (VRID 212.603.014) titulado: “Concepción: una tradición arquitectónica popular escondida detrás de las fachadas formales” (2012-2014)

Conflicto de intereses: El autor declara que no hay conflicto de intereses.

\section{Bibliografía}

Aguilera Rojas, J. (1994). Fundación de ciudades hispano-americanas. Madrid: Mapfre.

Alvarado, M.; Mege, P.; Báez, C. (eds.) (2001). Mapuche: fotografías siglos XIX y XX: construcción y montaje de un imaginario. Santiago de Chile: Pehuén Editores. http://www.memoriachilena.gob.cl/602/w3-article-79805.html

ANRED (2013). Neuquén: Incendiaron la ruka comunitaria del lof Campo Maripe https://www.anred.org/2013/08/31/neuquen-incendiaron-la-ruka-comunitaria-del-lof-campomaripe/

Aravena, A. (2002). Los mapuches-warriaches, procesos migratorios e identidad mapuche urbana en el siglo XX. En: G. Bocarra (Ed.), Colonización, resistencia y mestizaje en las américas, siglos XVI-XX (pp. 359-370). Lima, Perú: IFEA; Quito, Ecuador: Ediciones Abya-Yala.

Aravena, A. (2003). El rol de la memoria colectiva y de la memoria individual en la conversión identitaria mapuche. Estudios atacameños, (26), 89-96. DOl: http://dx.doi.org/10.4067/S0718$\underline{10432003002600010}$

Campos, F. (1985). Concepción en la primera mitad del siglo XX. Santiago: Museo Histórico Nacional.

Capellà Miternique, H. y Benedetto, A. (2006). Mendoza: La identidad a partir del reconocimiento de sus paisajes. Boletín de la GAEA, 18, 49-58. Recuperado de http://gaea.org.ar/contribuciones/CONTRIB06index.htm

Capellà Miternique, H. (2010). Des photos pour combattre l'oubli. Le destin tragique de Concepción (Chili). Géographies et culture, 73, 109-122. DOI: https://doi.org/10.4000/gc.1882

Capellà Miternique, H. (2013). The attachment as the culture of the place: Social resilience from the risk in Concepción (Chile). En S. Haruyama (Ed.), Coastal Geomorphology and Vulnerability of Disaster towards Disaster Risk Reduction (pp. 35-60). Tokio, Japón: TERRAPUB.

ACE, 15 (4.5) CC BY-ND 3.0 ES | UPC Barcelona, España | Las puertas de la memoria: Concepción (Chile).

DOI: http://dx.doi.org/10.5821/ace.15.45.9643 
Capellà Miternique, H. (2014). A vernacular architectonic tradition hidden behind formal façades: The case of Concepción in Chile. En M. Correia, G. Carlos \& S. Rocha (Eds.), Vernacular Heritage and Earthen Architecture: Contributions for Sustainable Development (pp. 711-716). CRC Press / Taylor \& Francis Group.

Carter, Th. (2005). Invitation to vernacular architecture: a guide to the study of ordinary buildings and landscapes. Knoxville, EE.UU.: University of Tennessee Press.

CHILECTRA (2001). Luces de modernidad: archivo fotográfico CHILECTRA. Santiago de Chile: Gerencia Corporativa de Comunicación Enersis S.A. Santiago de Chile: Larrea Impresores. http://www.memoriachilena.gob.cl/602/w3-article-74972.html

Cirlot, J.E. (1969). Diccionario de símbolos. Barcelona: Labor (2aㅡ Ed.).

Cirvini, S. (2011). Vernacular architecture in the Cuyo Region (Argentina). ACE: Architecture, City and Environment, 6(17), 15-36. DOI: https://doi.org/10.5821/ace.v6i17.2527

Clay, G. (1987). Right before your eyes, penetrating the urban environment. Washington, D.C., EE.UU.: Planners Press.

Conrad, J. (2010). Youth, heart of darkness, the end of the tether (Vol. 6). Cambridge, Reino Unido: Cambridge University Press.

Cooper, C.C. (1971). The house as symbol of self. No. 120. Institute of Urban \& Regional Development, University of California.

Cooper, J.C. (1978). An illustrated encyclopaedia of traditional symbols (Vol. 66). Londres, Reino Unido: Thames and Hudson.

Coquery-Vidrovitch, C y Georg, O. (1996). La ville européenne outre-mer: un modèle conquérant ? (XVèXXè). Paris, Francia: L'Harmattan.

Corraliza, J.A. (1987). La experiencia del ambiente. Percepción y significado del medio construido. Madrid, España: Tecnos.

Descola, P., y Pons, H. (2012). Más allá de naturaleza y cultura. Buenos Aires, Argentina: Amorrortu.

Eco, U. (1980). Function and sign: The semiotics of architecture. Signs, symbols and architecture, 1169.

Elizabeth, L. y Adams, C. (2005). Alternative construction: contemporary natural building methods. New Jersey, EE.UU.: John Wiley.

Francis, R. (1982). Symbols, images and social organization in Urban Sociology. The Sociological Review, 115-145. DOI: https://doi.org/10.1111/j.1467-954X.1982.tb00075.x

García Zarza, E. (1996). La ciudad en cuadrícula o hispanoamericana. Origen, evolución y situación actual. Salamanca, España: Universidad de Salamanca.

Hakim, B.S. (2007). Revitalizing Traditional Towns and Heritage Districts. ArchNet-IJAR: International Journal of Architectural Research 1.3: 153-166. Recuperado de https://archnet.org/publications/5089

Halbwachs, M. (1968). La Mémoire collective. Paris, Francia: Presses Universitaires de France (PUF).

Hays, S.P. (1993). From the History of the City to the History of the Urbanized Society. Journal of Urban History. 19: 3-25. DOI: https://doi.org/10.1177/009614429301900401

Kropff, L. (2004). 'Mapurbe': jóvenes mapuches urbanos. Kairos: Revista de Temas Sociales, 8(14), 1-12. Recuperado de http://www.revistakairos.org/mapurbe-jovenes-mapuches-urbanos/

ACE, 15 (4.5) CC BY-ND 3.0 ES | UPC Barcelona, España | Las puertas de la memoria: Concepción (Chile).

DOI: http://dx.doi.org/10.5821/ace.15.45.9643 
Kyvig, D.E. y Marty, M.A. (2000). Nearby history, exploring the past around you. Walnut Creek, California, EE.UU.: AltaMira Press.

Louvel, R. (2007). Crónicas y semblanzas de Concepción. Concepción, Chile: Sucesión René Louvel Bert.

Lynch, K. (1960). The image of the city. Cambridge, EE.UU.: MIT Press.

Meinig, D.W. y Jackson, J.B. (1979). The Interpretation of ordinary landscapes, geographical essays, New York, EE.UU.: Oxford University Press.

Mikesell, M.W. (1978). Tradition and innovation in cultural geography. Annals of the Association of American geographers, 68(1), 1-16. DOI: https://doi.org/10.1111/j.1467-8306.1978.tb01176.x

Nogué, J. (Ed.) (2007). La construcción social del paisaje. Madrid, España: Biblioteca Nueva.

Oliver, C. \& Zapata, F. (1950). Libro de Oro de la Historia de Concepción. Concepción, Chile: Editorial Litográfica.

Okhovat, H.; Almasifar, N. y Reza Bemanian, M. (2011). A research on historical and cultural buildings in iranian vernacular architecture. ACE: Architecture, City and Environment. 6(17), 37-58. DOI: https://doi.org/10.5821/ace.v6i17.2528

Pacheco, A. (1997). Historia de Concepción, Siglo XX. Concepción, Chile: Editorial Universidad de Concepción.

Pérez, L. y Espinoza, L. (2008). Planificación Urbana y Espacio Público en Concepción (1940-2004), Concepción: Editorial de la Universidad de Concepción.

Roderick L.R. (1983). Interpretation of vernacular architecture, a critical review. Vernacular Architecture. 14(1), 19-28. DOI: https://doi.org/10.1179/vea.1983.14.1.19

Salvalai, G.; Imperadori, M.; Lumina, F.; Mutti, E. y Polese, I. (2017). Architecture for refugees, resilience shelter project: a case study using recycled skis. Procedia engineering, 180, 1110-1120. DOI: https://doi.org/10.1016/j.proeng.2017.04.271

Sauer, C. (2008). The morphology of landscape. En T. Oakes y P.L. Price (Eds.), The cultural geography reader (pp. 108-116). Londres, Reino Unido: Routledge.

Savin, A. (1998). The Burden and the Treasure: Victor Perera's Sephardic Family Chronicle. Prooftexts. 18(3), 225-237. Recuperado de https://www.jstor.org/stable/20689520

Seike, T., Kim, Y., Hosaka, Y., Ida, S. Y Masuda, T. (2018). Intraregional reuse of emergency temporary housing in Japan. Sustainable Cities and Society, 42, 650-662. DOI: https://doi.org/10.1016/j.scs.2017.11.001

Solano, F. (1990). Ciudades hispanoamericanas y pueblos de indios. Madrid, España: CSIC.

Tuan, Yi Fu (1974). Topophilia: a study of environmental perception, attitudes, and values. New Jersey, EE.UU.: Prentice-Hall.

Tuan, Fu (1977). Space and Place: The Perspective of Experience. Minneapolis, MN, EE.UU.: University of Minnesota Press.

Villalobos, S. (2010). Estudios y ensayos en torno a la historia de Chile. Santiago de Chile, Chile: Editorial Bicentenario Universitaria.

Xiao-ju, Sahng \& Wu, Da-wei. (2009). Ecological evolution conception of the Inner Mongolia area residence. Shanxi Architecture, 34: 014.

ACE, 15 (4.5) CC BY-ND 3.0 ES | UPC Barcelona, España | Las puertas de la memoria: Concepción (Chile).

DOI: http://dx.doi.org/10.5821/ace.15.45.9643 